\title{
DISCOVERY OF TWO SUPERNOVAE IN THE NUCLEAR REGIONS OF THE LUMINOUS INFRARED GALAXY IC 883
}

\author{
E. Kankare ${ }^{1}$, S. Mattila ${ }^{1}$, S. Ryder ${ }^{2}$, P. Väisänen ${ }^{3}$, A. Alberdi ${ }^{4}$, A. Alonso-Herrero ${ }^{5,6}$, L. Colina ${ }^{5}$, A. Efstathiou ${ }^{7}$, \\ J. Kotilainen ${ }^{8}$, J. Melinder ${ }^{9}$, M.-A. Pérez-Torres ${ }^{4}$, C. Romero-CañIzales ${ }^{4}$, and A. TaKalo ${ }^{1}$ \\ ${ }^{1}$ Tuorla Observatory, Department of Physics and Astronomy, University of Turku, Väisäläntie 20, FI-21500 Piikkiö, Finland; erkki.kankare@utu.fi \\ ${ }^{2}$ Australian Astronomical Observatory, P.O. Box 296, Epping, NSW 1710, Australia \\ ${ }^{3}$ South African Astronomical Observatory, P.O. Box 9, Observatory 7935, South Africa \\ ${ }^{4}$ Instituto de Astrofsica de Andalucia, IAA-CSIC, Apartado 3004, 18080 Granada, Spain \\ ${ }^{5}$ Departamento de Astrofísica, Centro de Astrobiología, CSIC/INTA, Carretera de Torrejón a Ajalvir, km 4, 28850, Torrejón de Ardoz, Madrid, Spain \\ ${ }^{6}$ Instituto de Física de Cantabria, CSIC-Universidad de Cantabria, 39005 Santander, Spain \\ ${ }^{7}$ School of Sciences, European University Cyprus, Diogenes Street, Engomi, 1516 Nicosia, Cyprus \\ ${ }^{8}$ Finnish Centre for Astronomy with ESO (FINCA), University of Turku, Väisäläntie 20, FI-21500 Piikkiö, Finland \\ ${ }^{9}$ Department of Astronomy, Oskar Klein Centre, Stockholm University, AlbaNova University Centre, 10691 Stockholm, Sweden \\ Received 2011 October 25; accepted 2011 December 1; published 2011 December 15
}

\begin{abstract}
We report the discovery of two consecutive supernovae (SNe), 2010cu and 2011hi, located at 0!37 (180 pc) and 0.'79 $(380 \mathrm{pc})$ projected distance, respectively, from the center of the $K$-band nucleus of the luminous infrared galaxy (LIRG) IC 883. The SNe were discovered in an ongoing near-infrared $K$-band search for core-collapse $\mathrm{SNe}$ in such galaxies using the ALTAIR/NIRI adaptive optics system with laser guide star at the Gemini-North Telescope. These are thus the closest SNe yet discovered to an LIRG nucleus in optical or near-infrared wavelengths. The near-infrared light curves and colors of both SNe are consistent with core-collapse events. Both SNe seem to suffer from relatively low host galaxy extinction suggesting that regardless of their low projected galactocentric distances, they are not deeply buried in the nuclear regions of the host galaxy.
\end{abstract}

Key words: galaxies: individual (IC 883) - galaxies: starburst - infrared: galaxies - instrumentation: adaptive optics - supernovae: individual (SN 2010cu, SN 2011hi)

\section{INTRODUCTION}

Luminous $\left(L_{\mathrm{IR}}>10^{11} L_{\odot}\right)$ and ultraluminous $\left(L_{\mathrm{IR}}>\right.$ $10^{12} L_{\odot}$ ) infrared (IR) galaxies (LIRGs and ULIRGs, respectively) have high star formation (SF) rates. The fraction of SF in U/LIRGs in the local universe is small compared to that in normal spiral galaxies; however, at high redshift they become the dominant sources of SF (e.g., Magnelli et al. 2011). Stars more massive than $\sim 8 M_{\odot}$ explode as core-collapse supernovae $(\mathrm{CCSNe})$, and due to their short life cycles they are a very useful tool for tracing ongoing SF rates, independent of the conventional way of using the galaxy IR luminosity. Recently, Anderson et al. (2011) have presented evidence that the SN population in the interacting LIRG system Arp 299 differs from those observed in normal spiral galaxies with the Ib and IIb $\mathrm{SNe}$ being more numerous and centrally concentrated than more common Type II SNe. Until now, this kind of study has been impossible due to insufficient numbers of $\mathrm{SNe}$ discovered in $\mathrm{U} /$ LIRGs, providing a strong motivation for $\mathrm{SN}$ searches in galaxies with high SF rates. The extinction-free searches at radio wavelengths using very long baseline interferometry (VLBI) have been successful in discovering $\mathrm{SNe}$ and $\mathrm{SN}$ remnants in U/LIRGs, e.g., in Arp 220 (Parra et al. 2007; Batejat et al. 2011) and Arp 299 (Pérez-Torres et al. 2009; Ulvestad 2009). The reason for the low optical discovery rate of CCSNe in U/LIRGs is the high dust extinction combined with spatial concentration of $\mathrm{SF}$ in the crowded nuclear regions within the central few hundreds of parsecs (see, e.g., Soifer et al. 2001). Therefore, imaging in the near-IR where the extinction is strongly reduced and high spatial resolution is achievable with ground-based adaptive optics (AO) or space-based imaging is required. Recently, such methods have been shown to be very efficient with the discovery of several SNe in the LIRG nuclear/circumnuclear regions. SN 2004ip was discovered with the Very Large Telescope using the natural guide star NACO AO system (Mattila et al. 2007). SNe 2008cs and 2004iq reported in Kankare et al. (2008) were discovered using the Gemini-North Telescope with the laser guide star (LGS) assisted ALTAIR AO system and the Hubble Space Telescope (HST) NICMOS archive data, respectively.

In this Letter, we report the consecutive discovery of two $\mathrm{SNe}$ in the same host galaxy with the Gemini-North Telescope, ${ }^{10}$ SN 2010cu and SN 2011hi. Sanders et al. (2003) report the host LIRG IC 883 (also known as, e.g., UGC 8387, Arp 193, and IRAS $13183+3423$ ) to have an IR luminosity of $L_{\mathrm{IR}}=$ $L[8-1000 \mu \mathrm{m}]=4.7 \times 10^{11} L_{\odot}$ and a distance of $100 \mathrm{Mpc}$ $\left(\mathrm{H}_{0}=75 \mathrm{~km} \mathrm{~s}^{-1} \mathrm{Mpc}^{-1}\right)$ giving a projected linear distance scale $1^{\prime \prime}=485 \mathrm{pc}$. The IR luminosity of IC 883 indicates a CCSN rate of $\sim 1.3 \mathrm{yr}^{-1}$ derived with the empirical relation of Mattila \& Meikle (2001), consistent with the discovery of two SNe within two years. In C. Romero-Cañizales et al. (2012, in preparation) we report the details of our radio follow-up of $\mathrm{SNe}$ $2010 \mathrm{cu}$ and $2011 \mathrm{hi}$ and investigate the innermost nuclear regions of IC 883 through high-angular resolution, high-sensitivity radio observations.

\section{OBSERVATIONS AND RESULTS}

\subsection{Near-IR Observations}

SNe 2010cu and 2011hi were discovered as a result of our $K$-band search for highly obscured CCSNe in a sample of eight

\footnotetext{
10 Based on observations obtained at the Gemini Observatory, which is operated by the Association of Universities for Research in Astronomy, Inc., under a cooperative agreement with the NSF on behalf of the Gemini partnership: the National Science Foundation (United States), the Science and Technology Facilities Council (United Kingdom), the National Research Council (Canada), CONICYT (Chile), the Australian Research Council (Australia), Ministério da Ciência e Tecnologia (Brazil), and Ministerio de Ciencia, Tecnología e Innovación Productiva (Argentina).
} 

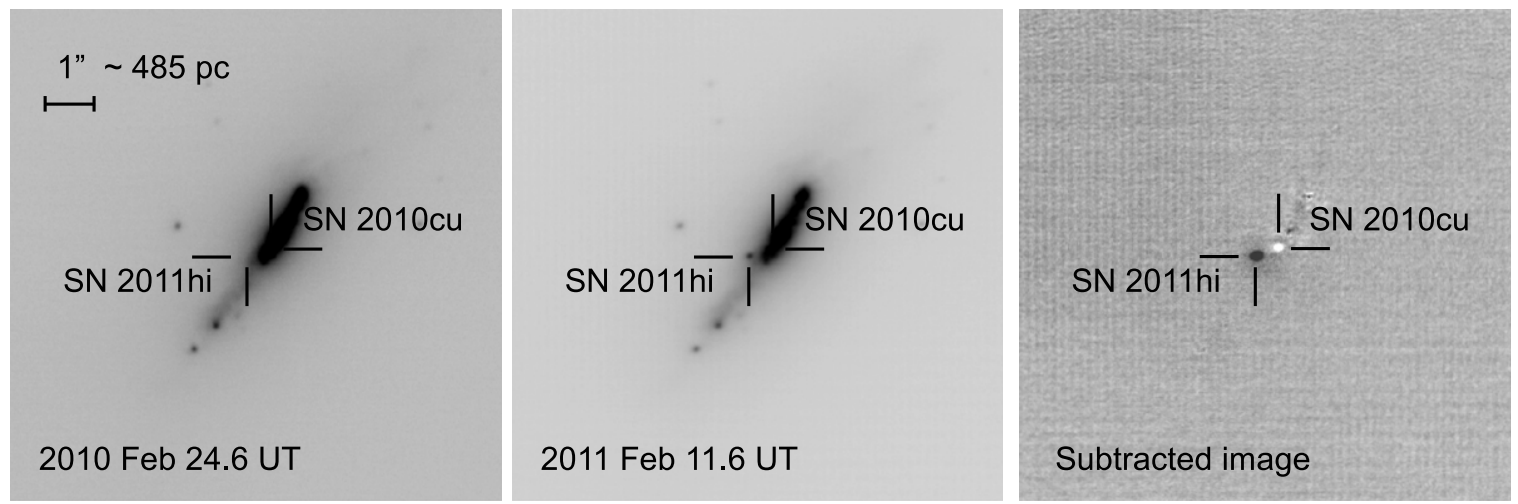

Figure 1. $10^{\prime \prime} \times 10^{\prime \prime}$ subsections of $K$-band Gemini ALTAIR/NIRI AO discovery images of SNe 2010cu (left panel) and 2011hi (middle panel) and the subtraction between these two (right panel). The smooth subtraction of the host galaxy IC 883 clearly demonstrates the good alignment and PSF match between the images and the two SNe can be clearly detected as individual point sources thanks to the high-angular resolution provided by the AO. North is up and east is to the left.

nearby LIRGs using the Gemini-North Telescope with the NearInfrared Imager (NIRI) with the ALTAIR LGS AO system $\left(0{ }^{\prime} 022\right.$ pixel $^{-1}$, FWHM $\left.\sim 00^{\prime \prime} 1\right)$. The individual NIRI images were reduced with IRAF using the NIRI package and registered and co-added using a bright field star as a reference. The discovery image of SN 2010cu was observed on 2010 February 24.6 UT (program GN-2010A-Q-40, PI: S. Ryder). Comparison to a previously obtained ALTAIR/NIRI $K$-band reference image from 2009 June 10.4 UT was done using a slightly modified version of the image subtraction package ISIS 2.2 (Alard \& Lupton 1998; Alard 2000). The software matches the point-spread functions (PSFs) and flux levels of a previously aligned pair of images by deriving an optimal convolution kernel. The image subtraction process revealed a new point source located only 0!37 from the brightest $K$-band core of IC 883 corresponding to a projected distance of $\sim 180 \mathrm{pc}$. The transient was reported to the Central Bureau for Astronomical Telegrams (CBAT) immediately after the discovery and was designated as possible supernova PSN K1002-1 (Kankare et al. 2010). A follow-up observation in $J H K$ on 2010 May 4.5 UT confirmed its SN nature resulting in a formal SN designation SN 2010cu (Ryder et al. 2010). Further follow-up observations were obtained on 2010 June 4.3 UT in HK and on 2011 February 11.6 UT in $J H K$ (GN-2011A-Q-48, PI: S. Ryder). As expected close to a year from discovery SN 2010cu had faded below the detection limit. However, a new point source had appeared 0.79 (projected distance $\sim 380 \mathrm{pc}$ ) from the $K$-band nucleus of the host galaxy. It was reported as a possible supernova (Kankare et al. 2011a) and follow-up observations in $J H K$ were obtained on 2011 April 18.5 UT, leading to the formal designation SN 2011hi (Kankare et al. 2011b). The $K$-band discovery images of SNe 2010cu and 2011hi and a subtraction between these two are shown in Figure 1. We note that the projected distance of SN 2010cu places it along the dusty ring reported by Clemens \& Alexander (2004), whereas SN 2011hi is located outside the ring.

Follow-up near-IR observations of SNe 2010cu and 2011hi were also attempted with the Nordic Optical Telescope ${ }^{11}$ (NOT) using the NOTCam instrument $\left(0^{\prime} .234\right.$ pixel $\left.^{-1}\right)$ on 2010 March 8.3 UT (seeing FWHM $\sim 0.7$ ) in HKs and 2011 February 20.2 UT (FWHM $\sim 0.9)$ in JHKs. However, neither epoch

\footnotetext{
11 Based on observations made with the Nordic Optical Telescope, operated on the island of La Palma jointly by Denmark, Finland, Iceland, Norway, and Sweden, in the Spanish Observatorio del Roque de los Muchachos of the Instituto de Astrofisica de Canarias.
}

provided detection of the SNe even with the help of image subtraction with the reference images obtained with the same instrument on 2009 March 15.1 UT (FWHM 1"1). This demonstrates the importance of high spatial resolution for SN searches in the nuclear regions of LIRGs.

Due to the small field of view (FOV) of the ALTAIR/NIRI AO setup of only $22 . .5 \times 22$ ".5 an accurate World Coordinate System (WCS) was derived with an iterative method. First, the WCS was derived for a Two Micron All Sky Survey (2MASS) $J$-band image of the field using 47 isolated point sources from the 2MASS catalog with astrometric accuracy of 0'.1 (Skrutskie et al. 2006). The 2MASS image was then aligned to an $H S T$ /Advanced Camera for Surveys $F 814 \mathrm{~W}$ image of the galaxy with a 3'.5 $\times 3$ '.5 FOV (obtained from the HST Science Archive) using eight sources common to both images. Finally, the HST image was transformed to the Gemini images using 14 point-like sources. Both transformation processes allowed shifts in $x$ and $y$, scaling and rotating. This yielded R.A. = $13^{\mathrm{h}} 20^{\mathrm{m}} 35.354$ and decl. $=+34^{\circ} 08^{\prime} 21^{\prime \prime} .86$ for SN 2010cu and R.A. $=13^{\mathrm{h}} 20^{\mathrm{m}} 35^{\mathrm{s}} .387$ and decl. $=+34^{\circ} 08^{\prime} 21^{\prime \prime} .69$ for SN 2011hi. For the brightest $K$-band core of IC 883 we obtain R.A. $=13^{\mathrm{h}} 20^{\mathrm{m}} 35^{\mathrm{s}} .336$ and decl. $=+34^{\circ} 08^{\prime} 22^{\prime \prime} .16$. The rms errors for the derived coordinates are 0.28 and $0{ }^{\prime \prime} 21$ for R.A. and decl., respectively. The alignment of the 2MASS image to match the HST image dominates the errors. The $K$-band core of IC 883 is within $1 \sigma$ from the $6.9 \mathrm{GHz}$ radio center reported in C. Romero-Cañizales et al. (2012, in preparation).

\subsection{Photometry and Extinctions}

The Gemini images were calibrated with an isolated field star in the FOV, 13203564+3408148 in the 2MASS catalog, using the standard star FS 131 (Hawarden et al. 2001) observations in $J H K$ obtained on 2011 April 18.5 UT. Both stars were measured with aperture photometry using Gaia and aperturecorrected to a radius of 100 pixel $\left(=2\right.$ '.2). This yielded $m_{J}=$ $17.44 \pm 0.02 \mathrm{mag}, m_{H}=16.77 \pm 0.02 \mathrm{mag}$, and $m_{K}=$ $16.50 \pm 0.02 \mathrm{mag}$ for the field star with the errors being dominated by the statistical photometric error. The photometry of SNe 2010cu and 2011hi was derived with the QUBA ${ }^{12}$ pipeline using the SNOOPY ${ }^{13}$ package, both running standard

\footnotetext{
12 Python package specifically designed for imaging and spectroscopy reduction and SN photometry in the IRAF environment developed by S. Valenti (Queens University, Belfast).

13 SNOOPY, originally presented in Patat (1996), has been implemented in IRAF by E. Cappellaro. The package is based on daophot, but optimized for SN magnitude measurements.
} 
Table 1

Photometry of SNe 2010cu and 2011hi

\begin{tabular}{lccc}
\hline \hline $\begin{array}{l}\text { JD } \\
(2400000+)\end{array}$ & $\begin{array}{c}m_{J} \\
(\mathrm{mag})\end{array}$ & $\begin{array}{c}m_{H} \\
(\mathrm{mag})\end{array}$ & $\begin{array}{c}m_{K} \\
(\mathrm{mag})\end{array}$ \\
\hline 55 & SN 2010cu \\
55252.1 & $\ldots$ & $\ldots$ & $17.36(0.06)$ \\
55321.0 & 19.7 & $19.18(0.09)$ & $18.74(0.15)$ \\
55351.8 & $\ldots$ & $19.52(0.05)$ & $19.24(0.09)$ \\
\hline 55604.1 & \multicolumn{5}{c}{ SN 2011hi } \\
55670.0 & $17.92(0.08)$ & $17.20(0.05)$ & $16.67(0.04)$ \\
& $18.07(0.06)$ & $17.00(0.05)$ & $16.42(0.04)$ \\
\hline
\end{tabular}

IRAF tasks. Three point sources in the ALTAIR/NIRI FOV were used to derive the PSF over the images used for fitting the SN in the subtracted images. Photometric errors were estimated by subtracting the fitted SN from the image and measuring several artificial sources simulated around the SN position with a dispersion of $1 \times$ FWHM. This error usually dominated over the statistical uncertainty of the PSF fitting. The $J$-band $5 \sigma$ upper limit for SN 2010cu was derived based on the faintest detected sources in the image taking into account the increased noise due to the image subtraction process. Table 1 lists apparent $\mathrm{SN}$ magnitudes, where errors are in parentheses.

The NOT images were also used for deriving the $J H K$ magnitudes of the ALTAIR/NIRI field star for a consistency check. Again, the QUBA pipeline was used and the following magnitudes obtained for the 2009 reference epoch, $m_{J}=$ $17.39 \pm 0.11 \mathrm{mag}, m_{H}=16.81 \pm 0.09 \mathrm{mag}$, and $m_{K}=$ $16.46 \pm 0.18 \mathrm{mag}$ using seven 2 MASS stars for the calibration.

As a simple approach to estimate the SN type and the host galaxy extinction in the lines of sight to SNe 2010cu and 2011 hi the $J H K$ light curve templates from Mattila \& Meikle (2001) were used. These templates are divided into two subtypes denoted "ordinary" (linearly declining) and "slowly declining" (based on Type IIL SN 1979C and Type IIn SN 1998S). The "ordinary" template contains a variety of SNe including Types Ib/c, IIL, and even IIb and IIP; despite some intrinsic differences in their near-IR light curves, we consider the template to describe at least the Type Ib/c SNe fairly well. The "slowly declining" template shows a near-IR excess originating from pre-existing dust in the circumstellar medium (CSM) of the progenitor (Fassia et al. 2000). The well-sampled near-IR light curves for the prototypical Type IIP SN 1999em from Krisciunas et al. (2009) were also used to represent the class of Type II SNe showing a plateau phase. From Schlegel et al. (1998) we obtain a Galactic extinction of $A_{V}=0.041$ for the IC 883 line of sight. For the host galaxy extinction both Cardelli et al. (1989) and Calzetti et al. (2000) reddening laws were considered and only the former for the Galactic extinction. We used $\chi^{2}$-fitting to derive the best fit for $V$-band host galaxy extinction $A_{V}$, the discovery epoch $t_{0}$ and the same constant shift $C$ for the absolute magnitude in all the three bands. For the "ordinary" and "slowly declining" templates $t_{0}$ is given relative to the $K$-band peak and for Type IIP with respect to the explosion date. The magnitude shift $C$ represents the intrinsic luminosity differences between the $\mathrm{SNe}$ and the templates. The $J$-band upper limit of SN 2010cu was not included in the fit. Results and reduced $\chi^{2}$ values are reported in Table 2 and the fits for each SN are shown in Figure 2.

From SN-free F110W and F222M images observed in an HST/NICMOS survey of LIRGs by Scoville et al. (2000) and obtained from the $H S T$ archive we produced a $J-K$ color map of the central regions of IC 883; see Figure 3. The NICMOS filter magnitudes were converted into Vega magnitudes using the transformations of Origlia \& Leitherer (2000). Assuming a typical intrinsic evolved stellar population color of $J-K \sim$ 0.9 mag (e.g., Fioc \& Rocca-Volmerange 1999) and the Calzetti extinction law the observed near-IR color of the galaxy can be converted into total line-of-sight extinction below the limit $\tau_{J} \sim 1.0$, i.e., with extinctions higher than $A_{V} \sim 3$ mag only the surface extinction is mapped and thus the values represent a lower limit.

As part of a future effort to estimate CCSN rates in our sample of LIRGs, detection efficiencies for the Gemini images were derived by simulating artificial $\mathrm{SNe}$ in the images. For this the images were divided into multiple contour regions based on the surface brightness, a reference frame was subtracted from the images using ISIS 2.2 and the subtracted frames were run through SExtractor (Bertin \& Arnouts 1996). Aperture photometry of the detected objects was compared with the standard deviation calculated for a random grid of aperture sums within each contour region. This procedure was repeated for different simulation magnitudes and the $3 \times$ standard deviation detection efficiency for each magnitude and contour area was calculated. Using s-curve (Dahlen et al. 2008) fitting to the results we derive a preliminary $50 \%$ detection efficiency for 18.3 mag sources in our $K$-band Gemini images in the innermost circumnuclear region in which both $\mathrm{SNe} 2010 \mathrm{cu}$ and 2011 hi were discovered. These initial results indicate that our search was sensitive enough to discover $\mathrm{SNe} \sim 1-1.5 \mathrm{mag}$ fainter than SNe 2010cu and 2011hi in the central regions of IC 883 (and LIRGs with similar properties). This corresponds to $\sim 10-15$ mag higher extinctions in $A_{V}$. However, our SN detection sensitivity drops significantly in the nuclear $<100 \mathrm{pc}$ regions (see also Romero-Cañizales et al. 2011) with only very

Table 2

Best $\chi^{2}$ Fits for the SN Parameters

\begin{tabular}{|c|c|c|c|c|c|c|c|c|}
\hline Template & $\begin{array}{c}A_{V} \\
(\mathrm{mag})\end{array}$ & $\begin{array}{c}t_{0} \\
\text { (days) }\end{array}$ & $\begin{array}{c}C \\
(\mathrm{mag})\end{array}$ & $\tilde{\chi}^{2}$ & $\begin{array}{c}A_{V} \\
(\mathrm{mag})\end{array}$ & $\begin{array}{c}t_{0} \\
\text { (days) }\end{array}$ & $\begin{array}{c}C \\
(\mathrm{mag})\end{array}$ & $\tilde{\chi}^{2}$ \\
\hline SN 2010cu & \multicolumn{4}{|c|}{ Cardelli Law } & \multicolumn{4}{|c|}{ Calzetti Law } \\
\hline Ordinary & 0.1 & -8 & +0.80 & 11 & 0.1 & -8 & +0.80 & 11 \\
\hline Slow & 0.0 & 6 & +2.20 & 8.5 & 0.0 & 6 & +2.20 & 8.5 \\
\hline IIP & 1.3 & 62 & +0.10 & 1.7 & 1.0 & 62 & +0.15 & 1.7 \\
\hline \multicolumn{9}{|l|}{ SN 2011hi } \\
\hline Ordinary & 6.8 & -38 & -1.35 & 5.0 & 4.7 & -38 & -1.00 & 4.4 \\
\hline Slow & 0.0 & 89 & -0.25 & 27 & 0.0 & 89 & -0.25 & 27 \\
\hline IIP & 7.0 & 31 & -1.65 & 9.4 & 5.0 & 31 & -1.30 & 8.8 \\
\hline
\end{tabular}



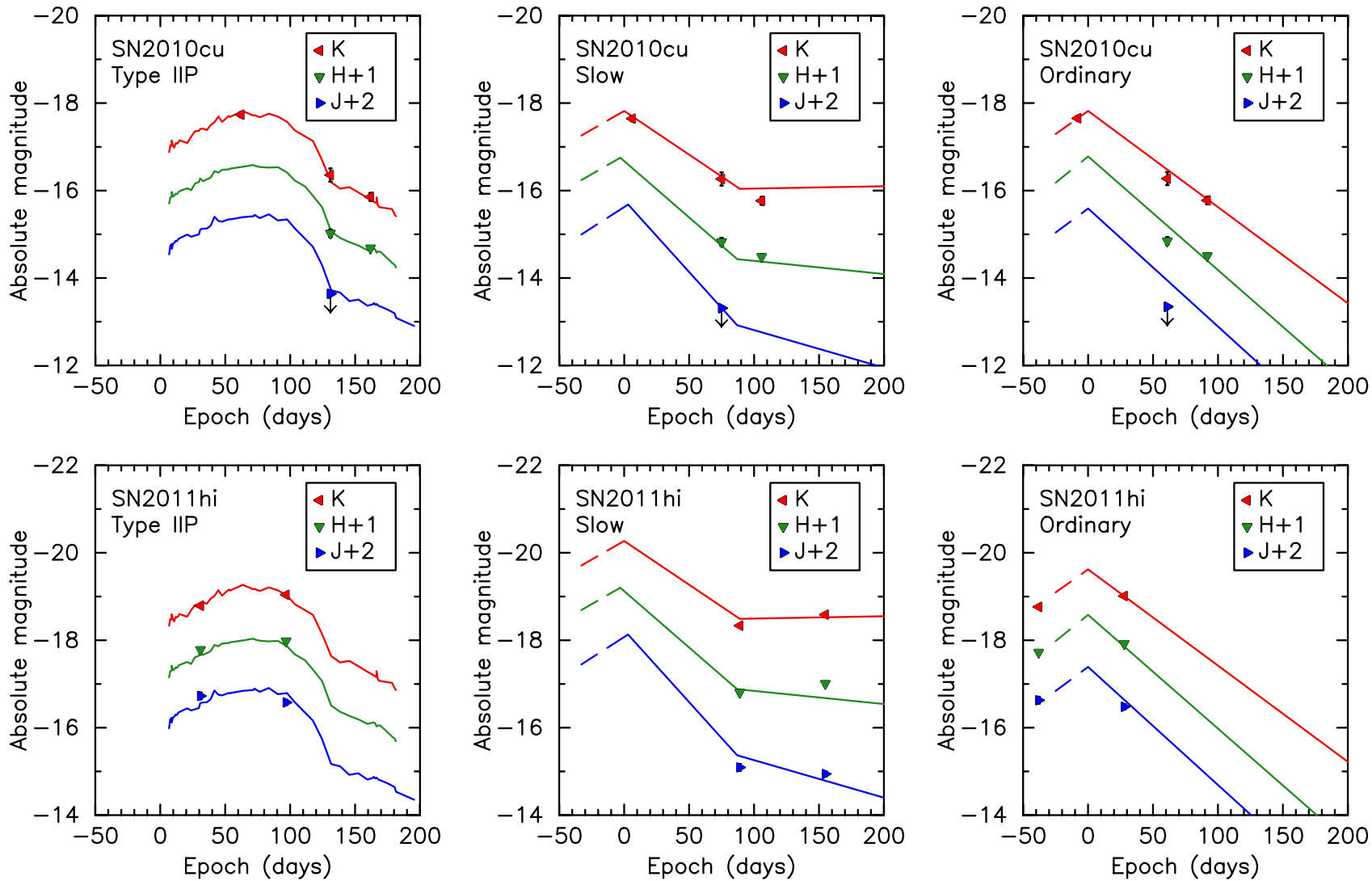

Figure 2. Template light curve fits for SN 2010cu and SN 2011hi are shown. The SN absolute magnitudes have been corrected for the derived total line-of-sight extinctions adopting the Calzetti extinction law. The template curves have been shifted in magnitude relative to the data by a constant $C$. The epoch for "slowly declining" and "ordinary" fits is relative to the $K$-band peak and for the Type IIP to the explosion date.

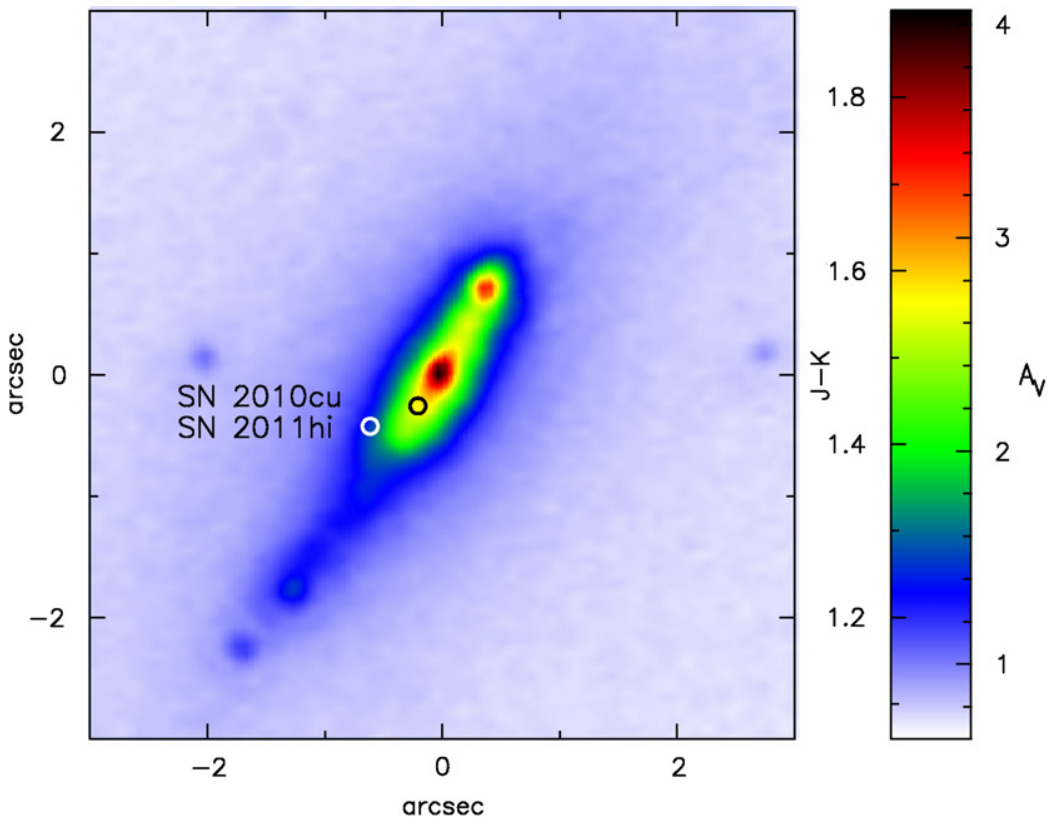

Figure 3. $6^{\prime \prime} \times 6^{\prime \prime} J-K H S T /$ NICMOS color map of the central regions of IC 883 in Vega magnitudes converted to extinction adopting the Calzetti extinction law. North is up and east is to the left.

high resolution VLBI observations being able to detect highly obscured SNe therein (e.g., Pérez-Torres et al. 2009).

\section{DISCUSSION}

For SN 2010cu we obtained satisfactory fits with both the "slowly declining" and Type IIP templates whereas the light curves and colors of the "ordinary" template fit are not consistent with the SN, when the $J$-band upper limit is taken into account. The absolute brightness and the discovery epoch of the SN at day 62 given by the Type IIP fit is also very reasonable given the fact that the galaxy was observed for the first time in over eight months. In contrast in the "slowly declining" template fit the SN 
would have to be underluminous by more than 2 mag compared to the template and discovered close to the maximum. However, based on only three epochs of photometry we cannot definitively determine the type of SN 2010cu between "slowly declining" and Type IIP. Regardless of the template used they both yield very low host galaxy extinction estimates $A_{V} \sim 0-1 \mathrm{mag}$.

For SN 2011hi we do not find the "ordinary" template fit very likely, even though it gives the best reduced $\chi^{2}$ value, as it would require the $\mathrm{SN}$ to be discovered on day -38 , thus having an unrealistically long rise time for an ordinary CCSN. We note that in Kankare et al. (2008) we found a $K$-band rise time of $>53$ days for $\mathrm{SN} 2008 \mathrm{cs}$ with the "slowly declining" template providing the best fit to the data. However, CCSNe interacting with their CSM have shown delayed rise to their near-IR peak compared to their optical discovery, e.g., SN 2006jc (Mattila et al. 2008). The "slowly declining" and the Type IIP templates both give reasonable fits and extinction estimates $A_{V} \sim 0-7 \mathrm{mag}$. In the Type IIP comparison the $\mathrm{SN}$ is expected to be $1.65 \mathrm{mag}$ brighter than the canonical SN 1999em; however, Type IIP SNe are known to show intrinsic variations of several magnitudes in their maximum brightness. For example, SN 2009kf (Botticella et al. 2010) was roughly 2 mag brighter than a prototypical Type IIP. Adopting the "slowly declining" template SN 2011hi would have been caught in the beginning of the near-IR excess phase, being intrinsically slightly brighter than the template. As only two epochs of photometry are available both template fits seem reasonable.

Radio observations of SNe 2010cu and 2011hi with the electronic European VLBI network (e-EVN) at $5 \mathrm{GHz}$ on 2011 March 23.1 UT and the electronic Multi-Element Remotely Linked Interferometer Network (e-MERLIN) at $6.9 \mathrm{GHz}$ resulted in upper limits for the radio emission from both $\mathrm{SNe}$ (see Canizales et al. 2011; C. Romero-Cañizales et al. 2012, in preparation, for details), which do not allow classifying them. In C. Romero-Cañizales et al. (2012, in preparation) an average SF rate of $185 M_{\odot} \mathrm{yr}^{-1}$ for IC 883 over the duration of the starburst was also derived based on the IR spectral energy distribution predicting a CCSN rate of $1.1 \mathrm{yr}^{-1}$, consistent with the discovery of two SNe within two years.

As shown earlier, the $J-K$ color map extinction of $\sim 3$ mag for the SN 2010cu line of sight through the whole galaxy is more of a lower limit for the total line-of-sight extinction. This gives freedom for any template fitted extinction estimate to be possible. However, the template fits for SN 2010cu give extinctions well below this limit. For SN 2011hi only the "slowly declining" template fit with negligible host galaxy extinction is found to be consistent with an estimated maximum line-of-sight extinction of $A_{V} \sim 1.5 \mathrm{mag}$ from the extinction map. However, higher localized extinction values, beyond the resolution of the extinction map, could be possible.

\section{CONCLUSIONS}

The AO assisted discovery of two consecutive SNe located at $<400 \mathrm{pc}$ from the LIRG nucleus demonstrates the importance of high spatial resolution in searches for CCSNe in highly crowded starburst regions in LIRGs. We note that of the five SNe discovered using high-resolution near-IR observations in our sample of LIRGs (see Section 1), four are within $1 \mathrm{kpc}$ from the host nucleus.

Based on the near-IR light curve fitting to templates we find SN 2010cu to be consistent with either a Type IIP or a near-IR bright Type IIn/L SN with a host galaxy extinction of $A_{V} \lesssim 1$ mag. Taking into account also the extinction map SN 2011 hi is found to be most consistent with a Type IIn/L SN during the near-IR excess phase with host galaxy extinction of $A_{V} \sim 0$ mag. A Type $\mathrm{Ib} / \mathrm{c}$ classification was found unlikely for both the SNe. Future near-IR and radio follow-up observations of SN 2011hi will be able to better constrain the nature and lineof-sight extinction of this potentially very interesting event.

The surprisingly low derived line-of-sight extinctions appear to be in conflict with the expectation of the occurrence of highly obscured CCSNe in the nuclear region of LIRGs. For example, in Kankare et al. (2008) $A_{V}=16$ mag of host galaxy extinction was derived with near-IR template light curve fitting for SN 2008cs, located at a projected distance of $1.5 \mathrm{kpc}$ from the host LIRG nucleus. Based on this it seems more likely that $\mathrm{SNe}$ 2010cu and 2011hi are located in the outer parts of the LIRG system which we see projected close to the LIRG nucleus. Additionally, the majority of LIRGs at the high IR luminosity end are complex interacting systems and their dust extinction distributions can be very complex compared to normal spiral galaxies with symmetric exponential disks; see, e.g., AlonsoHerrero et al. (2006) and Väisänen et al. (2008). Despite the low extinctions such SNe occurring close to the LIRG nuclei remain undiscovered by the current $\mathrm{SN}$ searches and will therefore contribute to the "SN rate problem" recently suggested by Horiuchi et al. (2011).

We thank the anonymous referee for useful comments and suggestions. E.K. acknowledges support from the Finnish Academy of Science and Letters (Vilho, Yrjö, and Kalle Väisälä Foundation). S.M. and E.K. acknowledge the support from the Academy of Finland (project: 8120503). A.A.H. and L.C. acknowledge support from the Spanish Plan Nacional de Astronomía y Astrofísica under grant AYA2010-21161-C02-1. A.A., M.A.P.-T., and C.R.-C. acknowledge financial support from the Spanish MICINN trough grant AYA2009-13036-CO201, co-funded with FEDER funds. J.K. acknowledges the support from the Academy of Finland (project: 2600021612).

\section{REFERENCES}

Alard, C. 2000, A\&AS, 144, 363

Alard, C., \& Lupton, R. H. 1998, ApJ, 503, 325

Alonso-Herrero, A., Rieke, G. H., Rieke, M. J., et al. 2006, ApJ, 650, 835 Anderson, J. P., Habergham, S. M., \& James, P. A. 2011, MNRAS, 416, 567

Batejat, F., Conway, J. E., Hurley, R., et al. 2011, ApJ, 740, 95

Bertin, E., \& Arnouts, S. 1996, A\&AS, 117, 393

Botticella, M. T., Trundle, C., Pastorello, A., et al. 2010, ApJ, 717, L52

Calzetti, D., Armus, L., Bohlin, R. C., et al. 2000, ApJ, 533, 682

Canizales, C. R., Perez-Torres, M. A., Alberdi, A., et al. 2011, ATel, 3291, 1

Cardelli, J. A., Clayton, G. C., \& Mathis, J. S. 1989, ApJ, 345, 245

Clemens, M. S., \& Alexander, P. 2004, MNRAS, 350, 66

Dahlen, T., Strolger, L.-G., \& Riess, A. G. 2008, ApJ, 681, 462

Fassia, A., Meikle, W. P. S., Vacca, W. D., et al. 2000, MNRAS, 318, 1093

Fioc, M., \& Rocca-Volmerange, B. 1999, A\&A, 351, 869

Hawarden, T. G., Leggett, S. K., Letawsky, M. B., Ballantyne, D. R., \& Casali, M. M. 2001, MNRAS, 325, 563

Horiuchi, S., Beacom, J. F., Kochanek, C. S., et al. 2011, ApJ, 738, 154

Kankare, E., Mattila, S., Ryder, S., et al. 2008, ApJ, 689, L97

Kankare, E., Mattila, S., Ryder, S., et al. 2010, CBET, 2213, 1

Kankare, E., Ryder, S., \& Mattila, S. 2011a, ATel, 3245, 1

Kankare, E., Ryder, S. D., Mattila, S., et al. 2011b, CBET, 2889, 1

Krisciunas, K., Hamuy, M., Suntzeff, N. B., et al. 2009, AJ, 137, 34

Magnelli, B., Elbaz, D., Chary, R. R., et al. 2011, A\&A, 528, A35

Mattila, S., \& Meikle, W. P. S. 2001, MNRAS, 324, 325

Mattila, S., Meikle, W. P. S., Lundqvist, P., et al. 2008, MNRAS, 389, 141

Mattila, S., Väisänen, P., Farrah, D., et al. 2007, ApJ, 659, L9

Origlia, L., \& Leitherer, C. 2000, AJ, 119, 2018

Parra, R., Conway, J. E., Diamond, P. J., et al. 2007, ApJ, 659, 314 
Patat, F. 1996, PhD Thesis, Univ. Padova

Pérez-Torres, M. A., Romero-Cañizales, C., Alberdi, A., \& Polatidis, A. 2009, A\&A, 507, L17

Romero-Cañizales, C., Mattila, S., Alberdi, A., et al. 2011, MNRAS, 415, 2688

Ryder, S., Kankare, E., \& Mattila, S. 2010, CBET, 2286, 1

Sanders, D. B., Mazzarella, J. M., Kim, D.-C., Surace, J. A., \& Soifer, B. T. 2003, AJ, 126, 1607
Schlegel, D. J., Finkbeiner, D. P., \& Davis, M. 1998, ApJ, 500, 525

Scoville, N. Z., Evans, A. S., Thompson, R., et al. 2000, AJ, 119, 991

Skrutskie, M. F., Cutri, R. M., Stiening, R., et al. 2006, AJ, 131, 1163

Soifer, B. T., Neugebauer, G., Matthews, K., et al. 2001, AJ, 122, 1213

Ulvestad, J. S. 2009, AJ, 138, 1529

Väisänen, P., Mattila, S., Kniazev, A., et al. 2008, MNRAS, 384, 886 